\title{
Psychologische Erkenntnisse zum Umgang mit komplexen Problemstellungen und zu Bedingungen kreativer Problem- \\ lösungen
}

\section{Joachim Funke}

Umgang mit komplexen Problemstellungen ist seit rund 25 Jahren zu einem Thema psychologischer Forschung geworden. Hatte man in der Denk- und Problemlöseforschung bis dahin den Fokus auf einfache Problemstellungen gelegt (zum Beispiel Denksportaufgaben, Streichholzprobleme), ist seit Mitte der 1970er Jahre eine Hinwendung der Forschung auf komplexe Probleme zu konstatieren. Der vorliegende Beitrag fasst in einem ersten Teil die wichtigsten Erkenntnisse psychologischer Forschung zum Umgang mit Komplexität zusammen. Wie sich dabei zeigen wird, sind bei komplexen Problemen kreative Lösungen gefragt. Daher widmet sich ein zweiter Teil den Erkenntnissen psychologischer Forschung zum Themengebiet der Kreativität. Ein abschließender dritter Teil versucht, praktische Empfehlungen auszusprechen.

\section{$\equiv 1$ Umgang mit komplexen Problemstellungen}

Dex Auftrag des Menschen, sich die Erde untertan $z u$ machen, hat soziale, wissenschaftliche, politische, technische und wirtschaftliche Entwicklungen ausgelöst, deren Schattenseiten uns heute zu überrollen drohen. Unsere Abhängigkeit von vielfältigen Infrastrukturen (Energie, Nahrung, Wasser, Information, etc.), die das tägliche Überleben sichern, ist so groß wie nie zuvor - man merkt es an der wachsenden Verletzlichkeit dieser filigranen Strukturen, die inzwischen weltweit gewebt sind. Von einem Sturm in Mittelamerika, der die Kaffeeernte bedroht, sind wir indirekt genauso betroffen wie vom Crash einer japanischen Bank, die zu Kursverlusten bei deutschen Aktienwerten führt. Mit fortschreitender Globalisierung nebmen die weltweiten Abhängigkeiten noch zu.

$\mathrm{Zu}$ Recht wird daher die Frage aufgeworfen, ob Menschen mit der von ihnen selbst geschaffenen Komplexität und deren Folgen umzugehen verstehen. Katastrophen im technischen, politischen, ökonomischen oder ökologischen Bereich weisen auf mögliche Grenzen menschlichen Umgangs mit Komplexität hin. Psychologische Forschung dazu stützt sich neben Beobachtungen und qualitativen Analysen solcher Phänomene zunehmend auch auf Experimente mit komplexen Unwelten. Diese werden in Form von "Mikrowelten" als computersimulierte Szenarien ins Labor geholt und erlauben dort unter kontrollierten Bedingungen eine Analyse des Geschehens (Brehmer \& Dörner, 1993). 
Als ein Beispiel sei hier die bekannte "Lohhausen"-Studie von Dörner, Kreuzig, Reither und Stäudel (1983) genannt. Der Name „Lohhausen" steht für die Simulation einer mitteleuropäischen Kleinstadt gleichen Namens, in der Versuchspersonen die Rolle des Bürgermeisters für einen Simulationszeitraum von 10 Jahren (verkürzt auf 10 Stunden Bearbeitungszeit) übernehmen sollten. Nach der bei Dörner (1981, S. 165) gegebenen Beschreibung hat die Stadt etwa 3500 Einwohner und lebt hauptsächlich von der stadteigenen Uhrenfabrik. Neben der Stadtverwaltung gibt es Arztpraxen, Einzelhandelsgeschäfte, eine Bank, Schulen, Kindergärten usw. Im Computermodell wurden nicht nur die ökonomischen Beziehungen abgebildet, sondern auch soziale, demokratische und psychologische Variablen (zum Beispiel Zufriedenheit der Einwohner). Versuchspersonen konnten in vielfältiger Weise in das System eingreifen: Sie konnten die Produktions- und Absatzpolitik der städtischen Fabrik beeinflussen, sie konnten Steuersätze ändern, Arbeitsplätze für Lehrer und Erzieherinnen schaffen, Arztpraxen einrichten und verpachten, Wohnungsbau betreiben, für Freizeiteinrichtungen sorgen usw. Das Computerszenario umfasste insgesamt rund 2000 Variablen.

Im Unterschied zu Fallstudien realer Entscheidungen konnten hier unter weitgehend kontrollierten Bedingungen im Labor die Entscheidungen einer Vielzahl von Personen beobachtet und registriert sowie mit anderen psychologischen Messungen in Zusammenhang gebracht werden. Außerdem erlaubt dieses Vorgehen die kontrollierte Variation von als wichtig erachteten Einflussgrößen (zum Beispiel Art des Feedbacks, Komplexität des Szenarios), um die Auswirkungen solcher Manipulationen festzustellen.

Bevor die wichtigsten Befunde aus Studien dieser Art in aller Kürze präsentiert werden, sollen die aus psychologischer Perspektive zentralen Merkmale komplexer Umwelten dargestellt werden.

\subsection{Merkmale von komplexen Umwelten und daraus resultierende Anforderungen}

Aus der Beschäftigung mit computersimulierten Mikrowelten haben sich fuinf Merkmale als wichtig zur Charakterisierung komplexer Umwelten erwiesen (vgl. Funke, 2003, Kap. 4). Sie sind generell zur Umschreibung komplexer Umwelten relevant und sollen daher hier vorgestellt werden.

\subsubsection{Komplexität}

Das naheliegendste Merkmal von komplexen Umwelten ist natürlich deren Komplexität. So unmöglich es scheint, eine verbindliche Definition dieses Begriffs zu liefern (siehe zum Beispiel Horgan, 1995; Klir, 1985), so einfach baben es sich manche Psychologen damit gemacht, indem sie sich nur auf die (große) Anzahl der in einem System vorhandenen Elemente oder Variablen bezogen haben. Wie auch immer die Begriffsbestimmung im Einzelnen ausfallen mag, sicher ist: Komplexität verlangt vom Handelnden den Einsatz komplexitätsreduzierender Verfahren wie etwa Abstraktion oder Vereinfachung durch Reduktion auf das Wesentliche.
Beispiel: Ein privater Umzug ist sicher weniger komplex als der Regierungsumzug von Bonn nach Berlin. Reduktion auf das Wesentliche heißt hier: Geplant wird auf einer gröberen Abstraktionsebene. Nicht für jedes einzelne Objekt wird eine Detailplanung gemacht.

\subsubsection{Vernetztheit}

Es kommt nicht nur auf die pure Anzahl von Elementen an, sondern auch auf deren Vernetzung. Je vernetzter die Elemente untereinander sind, umso mehr Abhängigkeiten entstehen. Vernetztheit verlangt vom Handelnden die Konstruktion eines Modells, das derartige Abhängigkeiten abbildet. Modellbildungssysteme sind hierfir nützliche Hilfsmittel (Lindig, ohne Jahrgang; Richmond, 2001; vgl. auch die Beiträge von Hub und Lindig in diesem Band).

Beispiel: Die Vernetztheit bei einem 1000-teiligen Puzzle fällt geringer aus als diejenige einer Uhr mit „nur" 500 Teilen, da bei letzterer eine große Zahl unterschiedlicher Funktionen mitbedacht werden muss. Ohne ein Modell dürften die Teile der Uhr kaum zusammensetzbar sein, die Puzzlestücke dagegen schon.

\subsubsection{Dynamik}

Komplexe Umwelten sind nicht statisch, sondern verändern sich über die Zeit hinweg, entweder aufgrund von Eingriffen in das System oder aufgrund vorhandener Eigendynamik. Dynamik fordert vom HandeInden die möglichst genaue Abschätzung zeitlicher Entwicklungsverläufe. Hierfür können Prognosemodelle herangezogen werden (vgl. Möbus \& Nagl, 1983).

Beispiel: Bei einer Hochwasser-Situation kann der Katastrophenstab die weitere Entwicklung gut vorhersagen, wenn der Wasserstand am Oberlauf und bei den $\mathrm{Zu}$ flüssen bekannt ist. Diese Dynamik macht Hochwasser-Lagen zu (unter dem Zeitaspekt) "harmlosen" Katastrophen im Unterschied etwa zu Explosionen, bei denen in kürzester Zeit folgenschwere Kettenreaktionen ausgelöst werden können. Viele Prozesse in der Natur weisen Eigendynamiken in Form von Wachstum oder Vergängnis auf.

\subsubsection{Intransparenz}

In komplexen Umwelten sind nicht immer alle benötigten Informationen verfigbar, sei es aus prinzipiellen oder aus zeitlichen Gründen. Intransparenz verlangt vom Handelnden einerseits ein Nachdenken darüber, welche Informationen noch beschafft werden könnten, andererseits werden Entscheidungen unter Unsicherheit verlangt. Dabei können einfache Heuristiken hilfreich sein (vgl. Gigerenzer \& Selten, 2001; Gigerenzer, Todd \& the ABC Research Group, 1999; vg1. Dieckmann \& Martignon in diesem Band). Heuristiken stellen Faustregeln dar, wie zum Beispiel „Entscheide Dich bei zwei Alternativen für diejenige, die Dir vertrauter vorkommt“. Dies sieht man etwa bei den abendlichen Quizsendungen, die zugleich demonstrieren, dass derartige Heuristiken keine Garantie auf die richtige Antwort liefern. Heuristiken kommen mit 
wenigen Informationen aus. Sie gelten als „fast and frugal“ (schnell und sparsam) und vereinfachen somit den Entscheidungsprozess.

\subsubsection{Polytelie (Vielzieligkeit)}

Komplexe Umwelten sind in aller Regel nicht hinsichtlich eines einzigen Kriteriums zu optimieren. Vielmehr stehen sich häufig konfligierende Ziele gegenüber. Vom Handelnden wird daher ein Ausgleich verschiedener Interessen in Form einer Kompromissbildung erwartet. In sozialen Kontexten stehen dafür heute Mediationstechniken zur Verfügung (vgl. Montada \& Kals, 2001).

Beispiel: Als Führungskraft in einem Unternehmen (zum Beispiel Lufthansa) muss man einerseits an eine gute Honorierung von Mitarbeitenden (zum Beispiel Piloten) denken, andererseits darf dies nicht so weit führen, dass kein Gewinn mehr abfällt oder die Unterschiede zu anderen Mitarbeitenden (zum Beispiel Kabinenpersonal) zu groß ausfallen.

\subsection{Komplexes Problemlösen: Eine Definition}

Vor dem Hintergnund dieser Merkmale wird der Forschungsgegenstand deutlicher: Komplexes Problemlösen erfolgt, um Barrieren zwischen einem gegebenen Ausgangs- und einem gewünschten Zielzustand durch Einsatz von kognitiven Tätigkeiten und Verhalten schrittweise zu reduzieren. Ausgangs- und Zielzustand wie auch die Barrieren erweisen sich als komplex, ändern sich dynamisch über die Zeit hinweg und können partiell intransparent sein. Die genauen Eigenschaften von Ausgangszustand, Zielzustand und Barrieren sind zu Beginn der Problemlösung unbekannt. Komplexes Problemlösen verlangt die effiziente Interaktion zwischen der problemlösenden Person oder Gruppe und den situativen Gegebenheiten, die sich aus der Aufgabenstellung ableiten lassen. Es verlangt den Einsatz von kognitiven, emotionalen und sozialen Ressourcen sowie von Wissen (nach Frensch \& Funke, 1995, S. 18).

\subsection{Zentrale Forschungsbefunde}

Die zahlreichen Befunde können hier nicht vollständig wiedergegeben werden (eine ausführlichere Darstellung findet man bei Funke, 2003, Kap. 5). Eingegangen werden soll auf ausgewählte Ergebnisse von zentraler Bedeutung.

\subsubsection{Zur Rolle der Intelligenz}

Zeigten die ersten Untersuchungen noch, dass die gemessene Intelligenz in geringem oder keinem Zusammenhang mit dem Lösen komplexer Probleme zu stehen schien (zum Beispiel Putz-Osterloh, 1981), ist nach heutigem Kenntnisstand von klaren Zusammenhängen zwischen dem Erfolg im Umgang mit einem komplexen Problem und einer spezifischen Intelligenzkomponente, der Verarbeitungskapazität, auszugehen (vgl. Süß, 1999). Verarbeitungskapazität wird durch Aufgaben zum formallogischen Denken sowie zu Analogien (,Finger verhält sich zu Hand wie Zeh zu?") erfasst und bildet am ehesten das ab, was frühe Intelligenzforscher wie Thurstone als ,reasoning" bezeichnet haben. Verarbeitungskapazität ist verwandt mit der Gedächtnisspanne, die eine Obergrenze der simultan za behaltenden Informationseinheiten im menschlichen Kurzzeitspeicher darstellt.

Tatsächlich erweist sich aber auch hohe Intelligenz nicht als Garant optimaler Problemlösungen. Dies mag damit zu tun haben, dass nach heutigen Intelligenzkonzeptionen (vgl. Funke \& Vaterrodt-Plünnecke, 1998) nicht nur analytische, sondern auch praktische und kreative Aspekte der Intelligenz unterschieden werden. Gemessen wird jedoch häufig nur ein Teilaspekt dieses mehrschichtigen Konzepts.

\subsubsection{Notfallreaktion des kognitiven Systems}

Diese Bezeichnung steht für ein Phänomen, das gerade bei scheiterndem Problemlösen in Erscheinung tritt: In derm Augenblick, in dem eine problemlösende Person die Anfordenungen nicht mehr bewältigen kann, kommt die enge Verzahnung von Kognition und Emotion besonders deutlich zum Vorschein. Dömer schildert das Beispiel einer Person, die in der „Lohhausen“-Simulation mit dem von ihr venursachten Niedergang der Kormmune überfordert war und sich statt mit wichtigen Themen mit dem Zustand der Parkbänke beschäftigt, um wenigstens hier noch Kompetenz demonstrieren zu können und ein positives Selbstbild zu bewahren (,Immerhin habe ich den Zustand der Bänke verbessert"). In dieser Notfallreaktion infolge eines mit Unlust einhergehenden Kontrollverlusts kommt es typischerweise zu einer Senkung des intellektuellen Niveaus, zu einer erhöhten Bereitschaft zu schnellem Handeln, einer degenerierten Hypothesenbildung (anstelle differenzierter Ursachenforschung wird zum Beispiel fälschlicherweise nur noch eine einzige Ursache als schuld an bestimmten Zuständen angesehen - zum Beispiel „die vielen Ausländer") und einer Entkonkretisierung von Zielen (siehe ausführlicher dazu Dörmer et al., 1983, S. 427). Entkonkretisierung von Zielen heißt zum Beispiel, dass man anstelle konkreter Zielsetzungen nur noch pauschal fordert: „Es muss etwas geschehen - so kann es nicht weitergehen". 1

Aus diesem Befund der engen Verzahnung von Kognition und Emotion sollte allerdings nicht der Schluss gezogen werden, dass Emotionen das Problemlösen in komplexen Situationen stören. Wie wir heute wissen, verändern Emotionen und Stimmungen die Art der Informationsverarbeitung (Fiedler, 1988, 2000): In positiver Stimmung entstehen kreative Ideen besser und es kommt zu einer globaleren Verarbeitung, während unter negativer Stimmung die analytische Verarbeitung von Details leichter fällt.

\subsubsection{Das Phänomen des "group think“}

Insbesondere in Situationen, in denen Gruppen von Personen ein Problem und dessen Lösung bearbeiten, ist das von Janis (1982) beschriebene Phänomen des Grup-

A Ähnlichkeiten mit parteipolitischen Aussagen sind rein zufällig ... 
pendenkens von Bedeutung. Dieses Gruppendenken charakterisiert er als „deteriotation of mental efficiency, reality testing, and moral judgement that results from ingroup pressures" (p. 9). Gerade Expertengruppen im politischen Bereich sind dafür besonders anfällig.

Beispiel Korea-Krise 1950-1953: Die Analyse der Korea-Krise (im Juni 1950 marschieren Truppen aus dem kommunistischen Nord-Korea in das pro-westlich gestimmte Südkorea ein, daraufhin beschließt die Truman-Administration den Einmarsch amerikanischer Truppen, die erfolglos am 38. Breitengrad kämpfen und am 19.7.1953 einem Waffenstillstand zustimmen, der die Nord-Süd-Grenze auf den 38. Breitengrad festlegt) verdeutlicht, dass in einer relativ homogenen BeraterGruppe nur wenig Alternativen generiert wurden. Durch eine einseitige Repräsentation des Problems (trotz der strategischen Bedeutungslosigkeit von Südkorea für die USA wird dort eine sowjetische Einflussnahme vermutet, der man aus prinzipiellen Gründen begegnen möchte) wird zudem nur eine einzige Lösung (nämlich die kriegerische Intervention) ernsthaft erwogen und dann auch realisiert. Die einmal getroffene Bewertung ,kommunistische Aggression“ legt allen Folgeentscheidungen eine Beschränkung auf, die über mehrere Jahre hinweg nicht mehr in Frage gestellt wird.

Das Streben nach Uniformität und Einhalten von Normen in der Gruppe erweist sich als abträglich für den Problemlöseprozess. Schaden nimmt dieser Prozess nach Janis in sieben Punkten: (1) Gruppenentscheidungen schränken den gesamten Raum der zu prüfenden Handlungsmöglichkeiten auf einige wenige Optionen ein; (2) die Gruppe überwacht ihre Ziele nicht richtig; (3) die Gruppe versäumt es, den von der Mehrheit favorisierten Lösungsweg kritisch zu bewerten; (4) Alternativen, die bereits am Anfang verworfen wurden, werden nicht wieder aufgegriffen; (5) Gruppenmitglieder holen nur selten Expertenrat von außerhalb ein; (6) Gruppenmitglieder verwenden wenig Energie darauf, Fakten oder Meinungen näher zu betrachten, die nicht ihren anfänglichen Präferenzen entsprechen; (7) die Gruppe verwendet wenig Zeit für die Beratung über Dinge, welche die geplanten Entscheiđungen verhindern könnten.

\subsubsection{Bedeutung von Feedback}

In Situationen mit dynamischen Anteilen, die sich aus Entwicklungen über die Zeit hinweg ergeben (etwa eine drohende Hochwasserkatastrophe), kommt dem Feedback über ablaufende Ereignisse besondere Bedeutung zu. Brehmer (1995) hat in experimentellen Untersuchungen zur Mikrowelt „Fire Fighting“, in der man als Versuchsperson einen an mehreren Stellen ausgebrochenen Waldbrand löschen sollte, verschiedene Arten von Verzögerungen differenziert: (a) Totzeiten, die aus der nicht unmittelbaren Umsetzung eines Einsatzbefehls resultieren; (b) Zeitkonstanten, die bei der Veränderung eines Systemszustands notwendig anfallen (zum Beispiel hört ein großes Feuer nicht sofort zu brennen auf, wenn es bewässert wird); (c) Informationsverzögenung, die sich aus verspäteten Rückmeldungen von Einsatzkräften ergeben.

Generell haben diese Feedbackverzögerungen starke negative Effekte auf das Lösen von komplexen Problemen in dynamischen Umwelten. Diese resultieren zum Beispiel daher, dass vorhandene Feedback-Effekte nur unvollständig oder falsch erkannt werden und damit die im System vorhandenen Effekte auf falsche Ursachen zurïckführen. Abhilfe schaffen hier Maßnamen, die so viel Verzögerungen wie möglich in einem System transparent machen. Dort, wo Totzeiten, Zeitkonstanten und Informationsverzögerungen unvermeidlich sind, sollten sie dem in solchen Situationen handelnden Akteur bewlisst gemacht werden.

\subsection{Zusammenfassende Bewertung}

Die Untersuchung komplexer Szenarien in denkpsychologischen Laboratorien hilft, die Ursachen des häufig unglücklichen Agierens von Menschen in derartigen Situationen besser zu verstehen. Falsch wäre es, von einer generellen Unfähigkeit von Menschen beim Umgang mit komplexen Problemen zu sprechen. Tatsächlich helfen Übung und Training, sowie Wissen über den Sachbereich als auch Wissen über menschliche Fehler in solchen Situationen, mit schwierigen Anfordenungen besser umzugehen (vgl. auch Buerschaper in diesem Band). Da die Forschung hierzu noch in den Anfängen steckt, werden sicher noch zahlreiche interessante Erkenntnisse aus dieser Forschung zu erwarten sein.

\section{Bedingungen kreativer Problemlösungen}

Da der menschliche Umgang mit komplexen Problemen -- wie wir gesehen haben mit Schwierigkeiten behaftet ist, kommt der Suche nach optimalen Bedingungen für kreative Problemlösungen besondere Bedeutung zu. Ganz generell kann gesagt werden, dass komplexe Problemstellungen kreative Problemlösungen erfordern. Daher werden nachfolgend in aller Kürze psychologische Erkenntnisse hierzu dargestellt, die sich auf die kreative Person, den kreativen Prozess, das kreative Produkt und das kreative Umfeld beziehen.

\subsection{Die kreative Person}

Muss man überdurchschnittlich intelligent sein, um kreative Leistungen hervorzubringen? Sternberg (1995) bejaht diese Aussage, fügt aber hinzu, dass oberhalb einer bestimmten Schwelle (ab einem IQ von 120) eine weitere Steigerung der Intelligenz keine Auswirkungen mehr auf Kreativität habe. Dabei ist ein Intelligenzkonzept unterstellt, das auf verschiedene Arten von Intelligenz (zum Beispiel analytische, praktische, kreative) abhebt und unterschiedliche Profile zulässt.

Hinsichtlich anderer Persönlichkeitseigenschaften zeigen sich bei kreativen Personen verstärkt Unabhängigkeit, Nonkonformismus, unkonventionelles Verhalten, weitgespannte Interessen, Offenheit für neue Erfahrungen, Risikobereitschaft sowie kognitive und verhaltensmäßige Flexibilität. Auch wenn zwischen Genie und Wahnsinn keine feste Verbindung besteht, können kreative Personen doch einen gewissen Grad psychopathologischen Verhaltens aufweisen, was für die Umwelt nicht immer angenehm ist. 
Die Annahme, dass kreative Leistungen vor allem im engen Zeitfenster zwischen dem 20. und 30. Lebensjahr erbracht würden, kann nicht gestützt werden. Je wissensintensiver der Bereich, umso mehr Erfahrung wird benötigt, und zahlreiche Faktoren können auch im höheren Lebensalter für kreative Produktivität sorgen, unter denen die Bereitschaft zur Auseinandersetzung mit geistigen Anforderungen zu den wichtigsten gehört. Wie das Beispiel Pablo Picasso zeigt, ist gerade dessen Alterswerk von einem Ausdruck geprägt, der viele Jugendwerke übertrifft. Auch in Literatur wie Politik finden sich viele Beispiele dafuir, dass gerade in höherem Lebensalter die „reiferen“" Werke geschaffen wurden. Der „Jugendwahn" manches Personalverantwortlichen erscheint angesichts solcher Sachverhalte geradezu dümmlich.

\subsection{Der kreative Prozess}

Kreative Denkakte erscheinen Außenstehenden gelegentlich als geniale Geistesblitze, die aus heiterem Himmel zu kommen scheinen. Dabei wird häufig die vielfach langjährige Vorarbeit übersehen, die hinter derartigen Spitzenleistungen steckt. Erfindungen, gerade technisch anspruchsvolle, entstehen nicht „einfach so“, sondern vor dem Hintergrund entsprechender Expertise oder Übung. Unter Expertise-Forschenden wird von Expertentum gesprochen, wenn jemand mindestens 10.000 Stunden in die Beschäftigung mit einem Gegenstand investiert hat (vgl. Gruber \& Mandl, 1998; Reimann, 1998).

Im Phasenmodell des kreativen Prozesse von Wallas (1926) entspricht dies der ersten Phase, die er Vorbereitung nannte. Die zweite Phase der Inkubation weist darauf hin, dass man gelegentlich die Beschäftigung mit einem Problem ruhen lassen kann, weil die „Infektion“ um die Metapher aufzugreifen bereits wirkt. In Zeiten der Nichtbeschäftigung ändern sich möglicherweise assoziative Verbindungen im Gehirn, die dann in der dritten Phase der Einsicht zu einer neuen Idee führen. Die Bewusstwerdung der schöpferischen Einsicht ist mit dem Begriff „Aha-Effekt" bezeichnet worden und beschreibt den Moment, wo die neue Idee die Schwelle zum Bewusstsein überschreitet. In der darauf folgenden vierten Phase setzt eine Bewertung ein, welche die neue Idee einer kritischen Beurteilung unterzieht. Wird diese Zensur überwunden, setzt die fünfte und letzte Phase der Ausarbeitung ein, da zum Beispiel von der Idee einer Glühbime bis zum ersten Prototyp noch ein weiter Weg $z \mathfrak{u}$ überwinden war. Hier sind noch einnal erhebliche Kräfte notwendig, um die kreative Idee Wirklichkeit werden zu lassen.

\subsection{Das kreative Produkt}

Was das Ereignis kreativen Denkens zu einem kreativen Produkt macht, ist - wie an der Diskussion über zeitgenössische Kunst festzustellen - nicht einfach zu sagen. Ob ein am Kopf zu befestigender Schirmaufsatz eine kreative Alternative zum traditionellen Regenschirm darstellt, ist nicht unumstritten. Auf jährlich stattfindenden Erfindermessen wird viel vorgestellt, was den Tag kaum überlebt.

In psychologischen Kreisen werden als Kriterien für ein kreatives Produkt dessen Neuigkeit und dessen Angemessenheit und Nützlichkeit im Sinne einer Problemlö- sung betrachtet. Dabei hängen diese Bewertungen vom Hintergrund des Beurteilers wie auch vom sozialen Konsens ab. Neben den beiden Hauptkriterien sind von Lubart (1994) noch drei Nebenkriterien genannt worden: Qualität, Bedeutung und Entstehungsgeschichte. Alle drei tragen zu einer differenzierten Bewertung kreativer Produkte bei; unser Respekt steigt, wenn wir etwa erfahren, dass das Produkt qualitativ ausgereift, sehr bedeutsam und zugleich Ergebnis langjähriger Entwicklung ist, wie zum Beispiel die Solarzelle.

\subsection{Das kreative Umfeld}

Nicht allein die kreative Person ist entscheidend - auch dem Umfeld kommt eine große Bedeutung zu. Künstler etwa sind auf den Austausch mit anderen Künstlern angewiesen, gleiches gilt für Literaten, Musiker oder Wissenschaftler. Die „peers" sind sowohl als Vorbilder wie als Kritiker ein wichtiger Teil des Umfelds. Aber auch soziokulturelle Einflüsse (,Zeitgeist") haben zu bestimmten historischen Epochen kreative Umfelder ausgemacht, wie ein Blick in die Geschichte zeigt. Simonton (1994) betont, dass - wie bereits im antiken Griechenland zu sehen sei - kulturelle Diversivität ein kreativitätsförderlicher Faktor, eine Ermutigung zu mehr Heterostatt Homogenität darstellt.

\subsection{Zusammenfassende Bewertung}

Kreativitätsforschung trägt zu einer Entmystifizierung eines Bereiches dar, in dem häufig erst das Ergebnis zu Kenntnis genommen wird und Staunen oder Ehrfurcht auslöst. Genauere Betrachtungen zeigen, was auf Seiten der Person an Vorbedingungen benötigt wird, wie der kreative Prozess abläuft und was ein kreatives Produkt auszeichnet. Auch đie Rolle des kreativen UmfeIds darf nicht unterschätzt werden. Auf einen Punkt gebracht: Als kreative Person wird man nicht geboren, sondern in jedem von uns stecken - eine gewisse Mindestintelligenz vorausgesetzt - kreative Potentiale. Diese hervortreten zu lassen, bedarf es allerdings einer Reihe von Umständen, die nicht immer gegeben sind.

\section{Praktische Empfehlungen}

Auch wenn es generell schwierig ist, aufgrund experimenteller Befunde im praxisnahen Setting zu generalisieren, sind doch einige Schlussfolgerungen nahe liegend und sollen am Ende vorgestellt werden. Die ersten beiden Punkte, Training und Feedback, beziehen sich schwerpunktmäßig auf einzelne Personen, die letzten beiden Punkte, Vermeidung von Gruppendenken und Schaffung kreativer Umwelten, schwerpunktmäßig auf die Ebene der Organisation. 
Ein Training zum Umgang mit komplexen Umwelten muss in allererster Linie Verständnis für die genannten Merkmale derartiger Situationen schaffen (vgl. auch Buerschaper in diesem Band). Zudem ist vielfältiges Training mit möglichst verschiedenen Mikrowelten dann hilfreich, wenn nicht einfach nur gespielt wird, sondern die im Zeitraffer ablaufenden Entscheidungen in der Rückschau kritisch reflektiert werden. Wichtige Leitfragen sind hier: Was war gelungen und warum? Was ist schief gegangen und warum?

Auch aktive Modellbildung (das heißt die formale Rekonstruktion eines Gegenstandsbereichs) scheint nützlich zu sein, haben doch die Konstrukteure von Mikrowelten häufig den größeren Lerneffekt als die Spieler dieser Szenarien. Als Modellbildner muss man sich mit den Abhängigkeiten und Dynamiken in einem System besonders gründlich und möglichst vollständig auseinandersetzen (siehe Senge \& Sterman, 1992).

Welche Arten von Training mit Hilfe von computersimulierten Szenarien sind zu unterscheiden? Dörner und Schaub (1992) differenzieren zwischen (a) der Vermittlung taktischer Fähigkeiten, (b) der Vermittlung von Wissen und Verständnis sowie (c) der Vermittlung strategischen Handelns.

\subsubsection{Vermittlung taktischer Fähigkeiten}

Hier geht es darum, sich möglichst konkret mit späteren Anforderungen auseinanderzusetzen und für bestimmte Situationen Routinehandlungen einzuüben. Typisches Beispiel hierfür sind Flugzeug-Simulatoren, an denen Piloten trainiert und auf Fehlersituationen vorbereitet werden (Gray, 2002). Gerade in Zeiten zunehmender Automatisienung spielt dieses Üben kritischer Situationen eine immer größere Rolle (Bainbridge, 1987). Wichtige Voraussetzung für den Erfolg solchen Taktik-Trainings ist die Übereinstimmung zwischen Simulator und Wirklichkeit (Hayes \& Singer, 1989). Je mehr allerdings Sensorik und Motorik an Stellenwert gewinnen, desto stärker ist man auf Training in der Realität angewiesen.

\subsubsection{Vermittlung von Wissen und Verständnis}

Während es beim gerade beschriebenen Bereich um den Erwerb konkreter Handlungsschritte ging, steht in diesem Bereich die Wissensaneignung durch Tun (,Learning by Doing", Anzai \& Simon, 1979; Kluwe, Misiak \& Haider, 1990; Schank, Berman \& Macpherson, 1999) im Vordergrund. Dabei geht es nicht um die passive Rezeption von Faktenwissen, sondern um den Erwerb von Verständnis über dynamische Zusammenhänge (etwa über das Wechselspiel in einem Räuber-Beute-Syștem oder über die Ausbreitung von AIDS). Auch die verschiedenen computergestützten Instrumente zur Modellbildung wie zum Beispiel Gamma (vgl. Hub, in diesem Band), Heraklit (vgl. Lindig, in diesem Band) oder STELLA (Richmond, 2001) können nach Senge und Sterman (1992) helfen, Eigenschaften dynamischer Systeme zu vermitteln.

\subsubsection{Vermittlung strategischen Handelns}

Der letzte Bereich ist der schwierigste, da hier Regeln für etwas gesucht werden, das kaum regelhaft abläuft (zum Beispiel politische und militärische, partiell auch wirtschaftliche Ereignisse) und wo winzige Unterschiede zu massiv unterschiedlichen Vorgehensweisen führen. Gerade hier können simulierte Szenarien natürlich einen Erfahrungsreichtum erzeugen, den man im realen Leben nie wird erreichen können. Gerade für diesen Bereich erweist sich der Szenario-Ansatz also als äußerst brauchbare Erfahrungsquelle.

Dennoch muss vor übertriebenen Hoffnungen gewarnt werden: Die hohe Kontextsensitivität kognitiver Aktivitäten macht einen direkten Transfer selbst zwischen ähnlichen Situationen eher unwahrscheinlich (Dörner \& Schaub, 1992; Funke, 1998; Strauß \& Kleinmann, 1995).

\subsection{Feedback}

Wie weiter oben dargestellt, sind gerade im Umgang mit komplexen Umwelten Feedback-Strukturen wichtig (siehe zum Beispiel van der Linden, Sonnentag, Frese \& van Dyck, 2001). Als Akteur in einem komplexen Geschehen braucht man Rückmeldungen über die Effekte seines Tuns. Bei zeitlich langsam verlaufenden Prozessen, zum Beispiel Entscheidungen über den Ausbau von Schulen und Hochschulen, die (vorhersehbar) vom Verlauf der Geburtenzahlen abhängen (Stichwort „Lehrerschwemme"), ist ein vorwärts gerichtetes Feedback („feedforward") zur Planung notwendig. Dieses Feedback sollte also in Form von Vorhersagen über zukünftige Zustände in einem Bereich geliefert werden (Prognosen). Bei zeitlich schnell verlaufenden Prozessen, zum Beispiel Entscheidungen im Rahmen eines Katastrophenmanagements, ist nachlaufende Kontrolle (,feedback") über die Wirkungen angeordneter Maßnahmen im Sinne einer Effektkontrolle notwendig. Checklisten helfen hierbei, die Gesamtlage im Blick zu behalten. Feedback ist ein essentieller Teil von Qualitätskontrolle und kann durch festgelegte Informationsflüsse sichergestellt werden. Wichtig ist nicht nur die Schaffung entsprechender Informationsflüsse, sondern auch das Festlegen von Konsequenzen bei Sollwertabweichungen. Das Nichtbeachten von Feedback zieht durchgängig negative Konsequenzen nach sich.

\subsection{Vermeidung von Gruppendenken}

Beim Umgang mit Komplexität sind die möglichst vielfältige Analyse einer Situation und die Diskussion von Zielen wünschenswert, um nicht zu simplifizierenden Bewertungen zu gelangen. Das Einholen einer zweiten Meinung ist nicht nur bei wichtigen ärztlichen Diagnosen empfehlenswert, sondern in all den Fällen, in denen wichtige und weit reichende Entscheidungen anstehen. Wichtig ist dabei die Unabhängigkeit der verschiedenen Meinungen. Die Kreativitätsforschung weist uns daher als Ausweg aus der Gefahr des Gruppendenkens die gezielte Suche nach Heterogenität und Diversität in der Zusammensetzung von Gruppen. Auch in modernen Zeiten braucht man also Hofnarren (Böhret, 1995). 


\subsection{Schaffung kreativer Umwelten}

Die Schaffung kreativer Umwelten stellt ein wichtiges Ziel von Organisationen dar, die mit komplexen und unberechenbaren Umwelten umgehen müssen und daher auf die Kreativität ihrer Mitglieder angewiesen sind. Kreativität ist nicht zu erzwingen - gerade extreme Anreize können den Tod kreativer Prozesse darstellen, da schöpferische Vorgänge nicht extrinsisch, sondem intrinsisch (,Ich will es wissen.") motiviert sind. Bedeutende Entdeckungen werden nicht gemacht, weil es den Nobelpreis als Anreiz gibt. Dies schließt nicht aus, dass kreative Leistungen belohnt werden, aber sie werden wohl kaum deswegen entstehen.

Gefahr droht kreativen Prozessen auch durch permanente Evaluation. Bewertungen können den vorschnellen Tod kreativer Ideen bewirken, weshalb Techniken wie „Brainstorming“" (vgl. Osborn, 1953) explizit auf Bewertungen verzichten, solange es um die Generierung neuer Ideen geht. Evaluationen und Bewertungsmaßstäbe neigen dazu, die Erfüllung von Normen und Konventionen zu honorieren, während Abweichungen negativ sanktioniert werden (Krampen \& Montada, 2002; Krampen, Montada \& Burkard, 2000)

3.5 Zusammenfassende Empfehlung: Handeln in komplexen Umwelten braucht kreative Problemlösungen

Ein zentrales Problem komplexer Umwelten ergibt sich aus der Schwierigkeit, Eingriffe in solche Systeme hinsichtlich ihrer Folgen abzuschätzen. Die Unberechenbarkeit zukünftiger Ereignisse wächst mit steigender Komplexität, Vernetztheit und Dynamik des Systems, um das es geht. So hilfreich Routinen und Automatismen im Einzelnen sein mögen: In den wirklich schwierigen Situationen versagt die Routine und es sind kreative Lösungen nötig. Hierfür müssen Vorbedingungen geschaffen werden, die sowohl auf Seiten der einzelnen Person wie auch auf Seiten der Organisation, in die eine Person eingebunden ist, das Entstehen von Kreativität möglich machen. Zugleich sollten kreativitätshemmende Faktoren (Konformismus, Druck, vorschnelle Bewertungen, etc.) so weit wie möglich reduziert werden. Diese Zielsetzung in die Tat umzusetzen ist deswegen so schwierig, weil sich Kreativität nicht anordnen lässt. Die zahlreichen Probleme von teilweise globalem Ausmaß machen dennoch alle Anstrengungen dazu unerlässlich - im Potential kreativer Problemlösungen liegt die Zukunft des Menschen.

\section{$4 \quad$ Literatur}

Anzai, K. \& Simon, H. A. (1979). The theory of learning by doing. Psychological Review, 86, 124-140. Bainbridge, L. (1987). Ironies of automation. In J. Rasmussen, K. Duncan \& J. Lepiat (Eds.), New technology and human error (pp. 271-283). Chichester: Wiley.

Böhret, C. (1995). Vom Hofnarten zum Politik-Coach. Zum Wiederaufstieg der persönlichen Beratung des Politikers. In C. Böhret \& M. Nowack (Hrsg.), Gesellschaftlich denken - kommunal handeln (S. 203-221). Mainz: AMV-Verlag.
Brehmer, B. (1995). Feedback delays in complex dynamic decision tasks. In P. A. Frensch \& J. Funke (Eds.), Complex problem solving: The European perspective (pp. 103-130). Hillsdale, NJ: Lawrence Erlbaum Associates.

Brehmer, B. \& Dörner, D. (1993). Experiments with computer-simulated microworlds: Escaping both the narrow straits of the laboratory and the deep blue sea of the field study. Computers in Human Behavior, 9, 171-184.

Dömer, D. (1981). Über die Schwierigkeiten menschlichen Umgangs mit Komplexität. Psychologische Rundschau, 32, 163-179.

Dömer, D., Kreuzig, H. W., Reither, F. \& Stäudel, T. (1983). Lohhausen. Vom Umgang mit Unbestimmtheit und Komplexität. Bern: Hans Huber.

Dörner, D. \& Schaub, H. (1992). Spiel und Wirklichkeit: Über die Verwendung und den Nutzen computersimulierter Planspiele. Kölner Zeitschrift für Wirtschaft und Pädagogik, 12, 55-78.

Fiedler, K. (1988). Emotional mood, cognitive style, and behavior regulation. In K. Fiedler \& J. Forgas (Eds.), Affect, cognition, and social behavior (pp. 100-119). Göttingen: Hogrefe.

Fiedler, K. (2000). Toward an integrative account of affect and cognitive phenomena using the BIAS computer algorithm. In J. P. Forgas (Ed.), Feeling and thinking: The role of affect in social cognition (pp. 223-252). Cambridge: Cambridge University Press.

Frensch, P. A. \& Funke, J. (Eds.). (1995). Complex problem solving: The European perspective. Hillsdale, NJ: Lawrence Erlbaum Associates.

Funke, J. (1998). Computer-based testing and training with scenarios from complex problem-solving research: Advantages and disadvantages. International Journal of Selection and Assessment, 6, 90-96.

Funke, J. (2003). Problemlösendes Denken. Stuttgart: Kohlhammer.

Funke, J. \& Vaterrodt-Plünnecke, B. (1998). Was ist Intelligenz? München: Beck.

Gigerenzer, G. \& Selten, R. (Eds.). (2001). Bounded rationality: The adaptive toolbox. Cambridge, MA: MIT Press.

Gigerenzer, G., Todd, P. M. \& the ABC-Research Group (Eds.) (1999). Simple heuristics that make us smart. New York: Oxford University Press.

Gray, W. D. (2002). Simulated task environments: The role of high-fidelity simulations, scaled worlds, synthetic environments, and laboratory tasks in basic and applied cognitive research. Cognitive Science Quarterly, 2, 205-227.

Gruber, H. \& Mandl, H. (1998). Das Entstehen von Expertise. In F. Klix \& H. Spada (Eds.), Enzyklopädie der Psychologie. Themenbereich C: Theorie und Forschung, Serie II: Kognition, Band 6: Wissen (pp. 583-615). Gottingen: Hogrefe.

Hayes, R. T. \& Singer, M. S. (1989). Simulation fidelity in training system design. Bridging the gap between reality and training. New York: Springer.

Horgan, J. (1995). Komplexitat in der Krise. Spektrum der Wissenschaft (9), 58-64.

Janis, I. L. (1982). Groupthink. Psychological studies of policy decisions and fiascoes. Revised and enlarged edition. Boston, MA: Houghton Mifflin

Klir, G. (1985). Complexity: Some general observations. Systems Research, 2, 131-140.

Kluwe, R. H., Misiak, C. \& Haider, H. (1990). Learning by doing in the control of a complex system. In H. Mandl, E. de Corte, N. Bennett \& H. F. Friedrich (Eds.), Learning and instruction. European research in an international context. Volume 2.1: Social and cognitive aspects of learning and instruction (pp. 197-218). Oxford: Pergamon Press.

Krampen, G. \& Montada, L. (2002). Wissenschaftsforschung in der Psychologie. Göttingen: Hogrefe

Krampen, G., Montada, L. \& Burkard, P. (2000). Evaluationskriterien für Forschung und Lehre an Psychologischen Instituten mit Hauptfachausbildung: Befunde einer Expertenberfragung in eigener Sache. In G. Krampen \& H. Zayer (Hrsg.), Psychologiedidaktik und Evaluation II (S. 133-142). Bonn: Deutscher Psychologen Verlag.

Lindig, R. (ohne Jahrgang). Vernetzt Denken und Handeln in der Projektarbeit mit dem Netzsimulator "HERAKLIT". Ein Leitfaden für die Projektabwicklung. Jena: Lindig Beratung (Selbstverlag).

Lubart, T. I. (1994). Creativity. In R. J. Sternberg (Ed.), Thinking and problem solving (pp. 290-323). San Diego, CA: Academic Press. 
Möbus, C. \& Nagl, W. (1983). Messung, Analyse und Prognose von Veränderungen. In J. Bredenkamp $\&$ H. Feger (Hrsg.), Hypothesenprüfung (Enzyklopädie der Psychologie, Themenbereich B: Methodologie und Methoden, Serie I: Forschungsmethoden der Psychologie, Band S) (S. 239-470). Göttingen: Hogrefe.

Montada, L. \& Kals, E. (2001). Mediation. Lehrbuch für Psychologen und Juristen. Weinheim: BeitzPVU.

Osbon, A. F. (1953). Applied imagination. New York: Scribner's.

Putz-Osterloh, W. (1981). Über die Beziehung zwischen Testintelligenz und Probiemlöseerfolg. Zeitschrif fü Psychologie, 189, 79-100.

Reimann, P. (1998). Novizen- und Expertenwissen. In F. Klix \& H. Spada (Hrsg.), Enzyklopädie der Psychologie, Themenbereich C: Theorie und Forschung, Serie II: Kognition, Band 6: Wissen (S. 335-365). Göttingen: Hogrefe.

Richmond, B. (2001). An introduction to systems thinking: Stella. Hanover, NH: High Performance Systems.

Schank, R. C., Berman, T. R. \& Macpherson, K. A. (1999). Learning by doing. In C. Reigeluth (Ed.) Instructional design theories and models (pp. 161-181). Mahwah, NJ: Lawrence Erlbaum Associates.

Senge, P. M. \& Sterman, J. D. (1992). Systems thinking and organizational leaming: Acting locally and thinking globally in the organization of the future. European Journal of Operational Research 59 137-150.

Simonton, D. K. (1994). Greatness: Who makes history and why. New York: Guilford Press

Sternberg, R. J. (1995). In search of the human mind. Fort Worth, TX: Harcourt Brace College Publishers.

Strauß, B. \& Kleinmann, M. (Hrsg.). (1995). Computersimulierte Szenarien in der Personalarbeit. Göttingen: Verlag für Angewandte Psychologie.

Süß, H.-M. (1999). Intelligenz und komplexes Problemlösen - Perspektiven für eine Kooperation zwischen differentiell-psychometrischer und kognitionspsychologischer Forschung. Psychologische Rundschau, 50, 220-228.

van der Linden, D., Sonnentag, S., Frese, M. \& van Dyck, C. (2001). Exploration strategies, performance, and error consequences when learning a complex computer task. Behaviour and Information Technology, 20, 189-198.

Voss, J. F., Wolfe, C. R., Lawrence, J. A. \& Engle, R. A. (1991). From representation to decision: An analysis of probiem solving in intemational relations. In R. J. Sternberg \& P. A. Frensch (Eds.), Complex problem solving: Principles and mechanisms (pp. 119-158). Hillsdale, NJ: Lawrence Erlbaum Associates.

Wallas, G. (1926). The art of thought. New York: Harcourt Brace.

\section{Einfache Heuristiken für gute Entscheidungen}

Anja Dieckmann und Laura Martignon

Ist es möglich, mit wenigen Informationen gute Entscheidungen zu treffen? Können einfache Entscheidungsregeln (Heuristiken) komplexen Aufgabenstellungen gerecht werden? Eine weit verbreitete Intuition ist, dass Entscheidungen besser werden, je mehr Informationen miteinander verrechnet werden. Wir werden in diesem Kapitel zeigen, dass oft das Gegenteil der Fall ist. Entscheidungen sind meist mit einem gewissen Grad an Ungewissheit behaftet - und gerade dann ist weniger häufig mehr. Die Fokussierung auf eine einzige oder wenige Variablen muss nicht nur keine Nachteile bringen, sondern kann die Vorhersagegenauigkeit sogar verbessern. Wir werden zunächst unseren Ansatz von anderen Rationalitätsauffassungen abgrenzen. Dann werden wir allgemein beschreiben, welche Qualitäten eine Heuristik auszeichnen. Die „Take The Best“-Heuristik und einfache Entscheidungsbäume werden dies konkret veranschaulichen. Aus den berichteten Befunden werden Schlussfolgerungen für Problemlöse- und Entscheidungsprozesse abgeleitet.

\section{Unterschiede zwischen einfachen Heuristiken und unbegrenzter Rationalität}

Ein Gedankenexperiment (Gigerenzer, im Druck) verdeutlicht den Unterschied zwischen unbegrenzter und eingeschränkter Rationalität in der jeweiligen Herangehensweise an Probleme. Daran lassen sich auch sehr gut unterschiedliche Strömungen in der Entscheidungsforschung aufzeigen.

Stellen Sie sich vor, ein Unternehmen möchte einen Roboter bauen, der Bälle fängt. Die Firmenleitung beauftragt vier konkurrierende Ingenieurteams mit dieser Aufgabe. Ein Team vertritt den klassischen Ansatz unbegrenzter Rationalität. Es versucht, die optimale Lösung zu finden, indem dem Roboter eine vollständige Repräsentation seiner Umwelt gegeben wird - und natürlich ein entsprechend leistungsfähiger Computer, der alle möglichen Umweltvariablen miteinander verrechnen kann. Da geworfene Bälle parabolische Wurfbahnen haben, wird dem Computer zunächst die Familie der Parabeln einprogrammiert. Um sich jeweils für die richtige Parabel zu entscheiden, braucht der Roboter zudem eine Vielzahl an Instrumenten, die die Entfernung des Abwurfpunktes, die Geschwindigkeit des Balls sowie den Abwurfwinkel messen. Hinzu kommt, dass eine Parabel nur eine Idealbeschreibung der Flugbahn darstellt - in der Realität müssen zusätzliche Faktoren wie Windrichtung und -geschwindigkeit berücksichtigt werden, die an verschiedenen Punkten gemessen und in die Berechnung einbezogen werden müssen. Letztendlich sollten, für bessere Vorhersagen, weitere Faktoren einkalkuliert werden, wie eine mögliche Drehung des Balls. Das Problem dieses Ingenieurteams ist nur, dass der Roboter so lange fuir die 
Rudolf Fisch

Dieter Beck (Hrsg.)

Komplexitäts-
management

Methoden zum Umgang mit komplexen Aufgaben-

stellungen in Wirtschaft, Regierung und Verwaltung 


\section{플}

VS Veriag für Sozialwissenschaften

Entstanden mit Beginn des Jahres 2004 aus den beiden Häusern

Leske+Budrich und Westdeutscher Verlag.

Die breite Basis für sozialwissenschaftliches Publizieren

Bibliografische Information Der Deutschen Bibliothek

Die Deutsche Bibliothek verzeichnet diese Publikation in der Deutschen Nationalbibtiografie

detaillierte bibliografische Daten sind im internet über <http://dnb.ddb.de> abrufbar.
1. Auflage Dezember 2004

Alle Rechte vorbehalten

VS Verlag für Soziaiwissenschaften/GWV Fachverlage $\mathrm{GmbH}$, Wiesbaden 2004

Lektorat: Frank Schindler/Dr. Tatjana Rollnik-Manke

Der VS vertag für Sozialwissenschaften ist ein Unternehmen von Springer Science+Business Media. www.vs-verlag.de
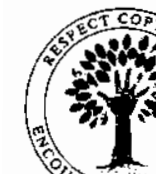

Das Werk einschließlich aller seiner Teile ist urheberrechtlich geschützt. Jede verwertung außerhaib der engen Grenzen des Urheberrechtsgesetzes ist ohne Zustimmung des verlags unzulässig und strafbar. Das gilt insbesondere Vervielfältigungen, Übersetzungen, Mikroverfilmungen und die Einspeicherung und verarbeitung in elektronischen Systemen.

Die Wiedergabe von Gebrauchsnamen, Handeisnamen, Warenbezeichnungen usw. in diesem Werk berechtigt auch ohne besondere Kennzeichnung nicht zu der Annahme, dass solche Namen im Sinne der Warenzeichen- und Markenschutz-Gesetzgebung als frei zu betrachten wären und daher von jedermann benutzt werden dürften.

Umschlaggestaltung: KünkelLopka Medienentwicklung, Heidelberg Druck und buchbinderische Verarbeitung. MercedesDruck Berlin fientrırkt aulf cäırofraiam und rhinfroi aohloirhtam oanior
Inhalt

Autorenverzeichnis

Vorwort

Rudolf Fisch und Dieter Beck

Handhabung komplexer Aufgabenstellungen in Organisationen -

Einführung und Übersicht...

\section{Psychologische Ansätze zur Handhabung komplexer Aufgaben}

Joachim Funke

Psychologische Erkenntnisse zum Umgang mit komplexen

Problemstellungen und zu Bedingungen kreativer Problemlösungen

Anja Dieckmann und Laura Martignon

Einfache Heuristiken für gute Entscheidungen

\section{Unterstützende Verfahren und Bedingungen ihres Einsatzes}

Dieter Beck

Übersicht über Verfahren zum Umgang mit komplexen

Aufgabenstellungen..

Rolf Bronner

Planungs- und Entscheidungsmethoden zur Komplexitätshandhabung und Rationalitätssicherung.

Heinrich Reinermann

Zum Einsatz von Verfahren und Werkzeugen der Entscheidungsunterstützung - Eine kritische Betrachtung .

Dietrich Fürst

Rahmenbedingungen des Einsatzes von Planungsverfahren

im öffentlichen Sektor

\section{Ausgewählte Verfahren zur Handhabung komplexer Aufgaben}

Frederic Vester

Vernetztes Denken und Handeln als Ansatz zur Krisenbewältigung

Gabriele Harrer

Das Vester'sche Sensitivitätsmodell .

Reinhard Lindig

Vernetzt Denken und Handeln bei komplexen Aufgabenstellungen 


\section{Hanns $\mathrm{Hub}$}

GAMMA als Methode und Werkzeug zur Bearbeitung komplexer

Aufgabenstellungen

Ralph Becker und Barbara Wöhler

Strategieumsetzung mit der Balanced Scorecard - Theorie und Praxis

\section{Praxisbeispiele}

Klaus König

Komplexitätsbewältigung in Regierungszentralen -

Ein Erfahrungsbericht.

Helmut Albert

Komplexe Aufgaben der Nachrichtendienste im Rahmen der

staatlichen Gefahrenabwehr - Organisation und Arbeitsweise

der Verfassungsschutzbehörden in Deutschland

Arjen Boin und Paul 't Hart

Coping with crisis complexity: Trends, challenges and pathways

Hans-Joachim Fietkau

Komplexitätsgestaltung in Mediationsverfahren.

Hans-Peter Meister und Christopher Gohl

Politische Mediation bei umstrittenen Infrastrukturprojekten -

Das Beispiel des Frankfurter Flughafens

Petra Badke-Schaub

Zwischen Kreativität und Systematik - Eine kognitions- und sozial-

psychologische Analyse des Einsatzes von Problemlösetechniken in der

Produktentwicklung

\section{Praktische Konsequenzen}

Comelius Buerschaper

Komplexität managen - Trainingsansätze und Nutzen

Rudolf Fisch und Dieter Beck

Was tun angesichts komplexer Aufgaben?

Stichwortverzeichnis

\section{Autorenverzeichnis}

Dr. Felmut Albert, Landesamt fuir Verfassungsschutz des Saarlandes, Postfach 102063, 66020 Saarbrücken.

Prof. Dr. Petra Badke-Schaub, Faculty of Industrial Design Engineering, TU Delft, Landbergstraat 15, 2628 CE Delft, The Netherlands.

Priv.-Doz. Dr. Dieter Beck, Forschungsinstitut für öffentliche Verwaltung bei der Deutschen Hochschule für Verwaltungswissenschaften Speyer, Postfach 1409, 67324 Speyer.

Dr. Ralph Becker, Horváth \& Partner GmbH, Rotebühlstraße 121, 70178 Stuttgart.

Prof. Dr. Rolf Bronner, Fachbereich Rechts- und Wirtschaftswissenschaften, Johannes Gutenberg-Universität Mainz, Saarstraße 21, 55099 Mainz.

Dr. Arjen Boin, Department of Public Administration, Crisis Research Center, Leiden University, P.O. Box 9555, NL-2300 RB Leiden, The Netherlands.

Cornelius Buerschaper, Dipl.-Psych., Wöhlertstraße 12, 10115 Berlin..

Anja Dieckmann, Dipl.-Psych., Max-Planck-Institut für Bildungsforschung, Lentzeallee 94, 14195 Berlin.

Dr. Hans Joachim Fietkau, Wissenschaftszentrum Berlin für Sozialforschung GmbH, Reichpietschufer 50, 10785 Berlin.

Prof. Dr. Rudolf Fisch, Deutsche Hochschule fuir Verwaltungswissenschaften Speyer, Postfach 1409, 67324 Speyer.

Prof. Dr. Dietrich Fürst, Institut für Raumplanung und Raumforschung, Universität Hannover, Westermannweg 35, 30419 Hannover.

Prof. Dr. Joachim Funke, Psychologisches Institut, Ruprecht-Karls-Universität Heidelberg, Hauptstraße 47-51, 69117 Heidelberg.

Christopher Gohl, M.A., IFOK GmbH - Institut für Organisationskommunikation, Berliner Ring 89, 64625 Bensheim.

Gabriele Harrer, Dipl.-Geol., Frederic Vester GmbH, Nussbaumstraße 14, 80336 München.

Prof. Dr. Paul 't Hart, Utrecht School of Governance, University of Utrecht, Bijlhouwerstraat 6, NL-3511 ZC Utrecht, The Netherlands.

Prof. Dr. Hanns Hub, Hub Consulting, Rathausstraße 11/2, 72660 Beuren 